\title{
On the universal spectrum of periods in the time series of temperature fluctuations in starlings and rats
}

\author{
Diatroptov M.E. ${ }^{1}$, Panchelyuga V.A. ${ }^{2}$, Panchelyuga M.S. ${ }^{2}$, Seraya O.Yu. ${ }^{2}$ \\ victor.panchelyuga@gmail.com \\ ${ }^{1}$ A.N. Severtsov Institute of Ecology and Evolution, RAS, Moscow, Russia; \\ ${ }^{2}$ Institute of Theoretical and Experimental Biophysics, RAS, Pushchino, Russia
}

\begin{abstract}
The paper considers a spectral analysis based on Fourier transform of the time series in the temperature fluctuations in the bodies of common starlings (Sturnus vulgaris) and rats (mature Wistar males). The spectra of the periods in the starlings and rats contain the same sets of harmonics, so on this basis we can tell about a common spectrum. Interesting to note that the spectrum coincides with a previously revealed spectrum of the periods, obtained with the use of local fractal analysis by the all permutations method in the course of studies of time series of the alpha decay rate fluctuations. Despite different methods of the experimental data processing used in this work (spectral analysis based on Fourier transform and local fractal analysis by the all permutations method), as well as different raw experimental data (the temperature fluctuations and alpha-decay rate fluctuations), the same spectrum of periods was obtained. On this basis, we can consider the spectrum of periods as universal one.
\end{abstract}

Keywords: starlings; rats; temperature fluctuations; universal spectrum, alfa-decay; local fractal analysis; all permutations method

\section{Introduction}

The papers [1-2] present the studies of the time series in the alfa-decay rate fluctuations. The studies were performed with the local fractal analysis by all permutations method [3-4], and produced the following results: 1) the authors have demonstrated the presence of a stable spectrum of periods in the analyzed time series; 2) the periods observed in the fluctuations of the alfa-decay rate coincided with the periods of the Earth natural oscillations (ENOs);3) the authors have demonstrated that the periods revealed in the time series of various nature processes coincide with the periods in the spectrum of the alfa-decay fluctuations. Basing on 3), a factor causing the periods in the fluctuations of the alfa-decay rate can be considered as a universal underlying condition, its biological "activity" being of special interest.

The biological periods coinciding with the periods revealed in the spectrum of the radioactive decay rate fluctuations were found in the motion behavior of the experimental animals [5], in the time series of the fluctuations of the R-R-intervals [6], at the investigation of the dynamic dielectric constant of biological object (Blattella germanica) [7], etc.

Unfortunately, few studies of the biological systems include thorough investigations of the periods that fall into the range from several minutes to tens of minutes. This may be explained by the fact that somewhat apparent alterations in controlled biological parameters usually take time from tens of minutes to hours, so a measurement interval of a minute or less that provides a spectrum within a range of minutes-tens-minutes is considered as a less informative and hence used rarely. The aim of the present study is to fill this gap.

\section{Raw experimental material}

Four starlings (Sturnus vulgaris) (two males and two females) were used to study the dynamics of the body temperature in birds. The birds were kept in the individual cages $35 \times 40 \times 30 \mathrm{~cm}$ at natural light.

The second part of the study was performed with the mature Wistar males $(n=14)$ of $280-320 \mathrm{~g}$ body mass. The animals were kept in the plastic cages $(60 \times 18,5 \times 38 \mathrm{~cm}), 1$ 2 animal per a cage at permanent artificial lighting from a source with $4200 \mathrm{~K}$ color temperature and 1200-1500 lx intensity. The animals were distributed into three experimental groups: I - intact animals $(n=5)$, II gonadectomized $(n=6)$ and III - animals with the combined surgical gonadectomy and adrenalectomy $(n=3)$. During the surgeries, the animals were anaesthesized with the intramuscular Zoletil («Virbac Sante Animale», France), $10 \mathrm{mg} / \mathrm{kg}$ of body mass.

The body temperature of starlings and rats was measured with the intraperitoneally implanted sensors DTN3-28 («EMBI RESEARCH», Novosibirsk) programmed to measure temperature once a minute in the starlings and every other minute in rats.

The most probable hormones determining the ultradian rhythmical recurrence in mammals are glucocorticosteroids produced by the adrenals and, to a lesser extent, by reproductive hormones. The ultradian variability of the secretion of the hypothalamic-pituitaryadrenal axis hormones can be explained by the presence of an oscillator controlled by the central nervous system and the mechanisms of the primary feedback. In such a way, the analysis of the alterations of the parameters of ultradian rhythms in the rats with the extirpated adrenals and testes can give a possibility to clarify the effect of the negative feedback on the formation of these biorhythms.

Fig. 1 shows an example of the experimental registration: one time series of a gonadectomized rat temperature measurements with an intraperitoneally implanted sensor DTN3-28. Measurement interval is 2 $\min$. 


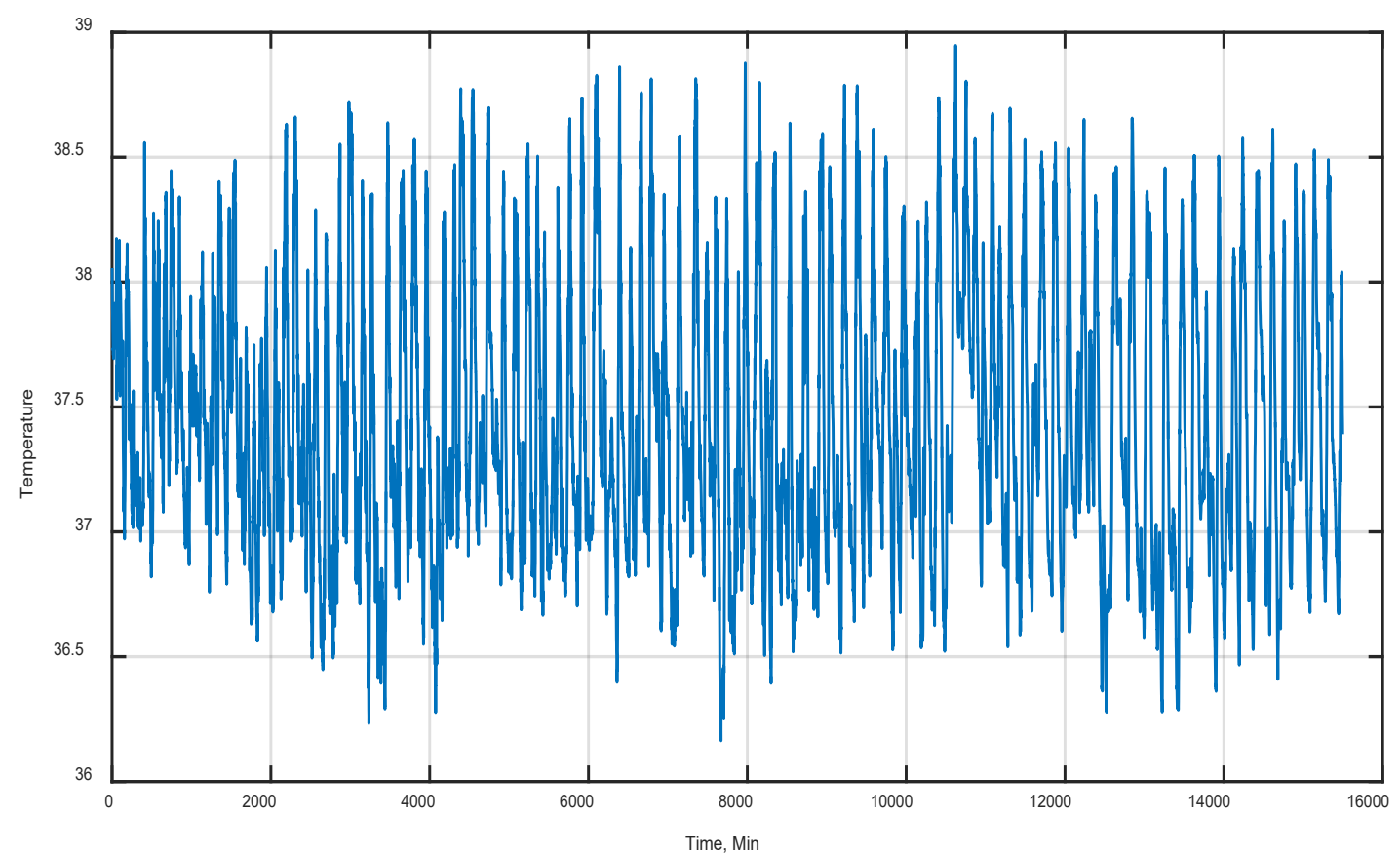

Fig. 1. Time series of rat temperature fluctuations

\section{Results. Comparison of spectra}

We constructed the power spectra for the time series similar to that shown at Fig. 1, the values of the spectra were summarized for the appropriate frequencies in each individual spectrum belonging to one of the groups: 1) starlings, 2) I - intact rats, 3) II - gonadectomized rats and 4) III - rats with the surgical gonadectomy and adrenalectomy. In such a way, we have obtained four power spectra. Fig. 2 presents an example of them: a power spectrum for the rats group III. A rectangular insert near each peak shows its period (X-coordinate) in minutes. All values of the periods shown at Fig.2 are recorded in an appropriate line of Table 1. Fig. 3 shows an enlarged portion of the spectrum presented at Fig.2 for the period range of up to $24 \mathrm{~min}$. The spectrum is four times smoothed with a running average procedure with the use of the Poisson triangular window. The values of the periods shown at Fig.3 are included into Table 1 too.

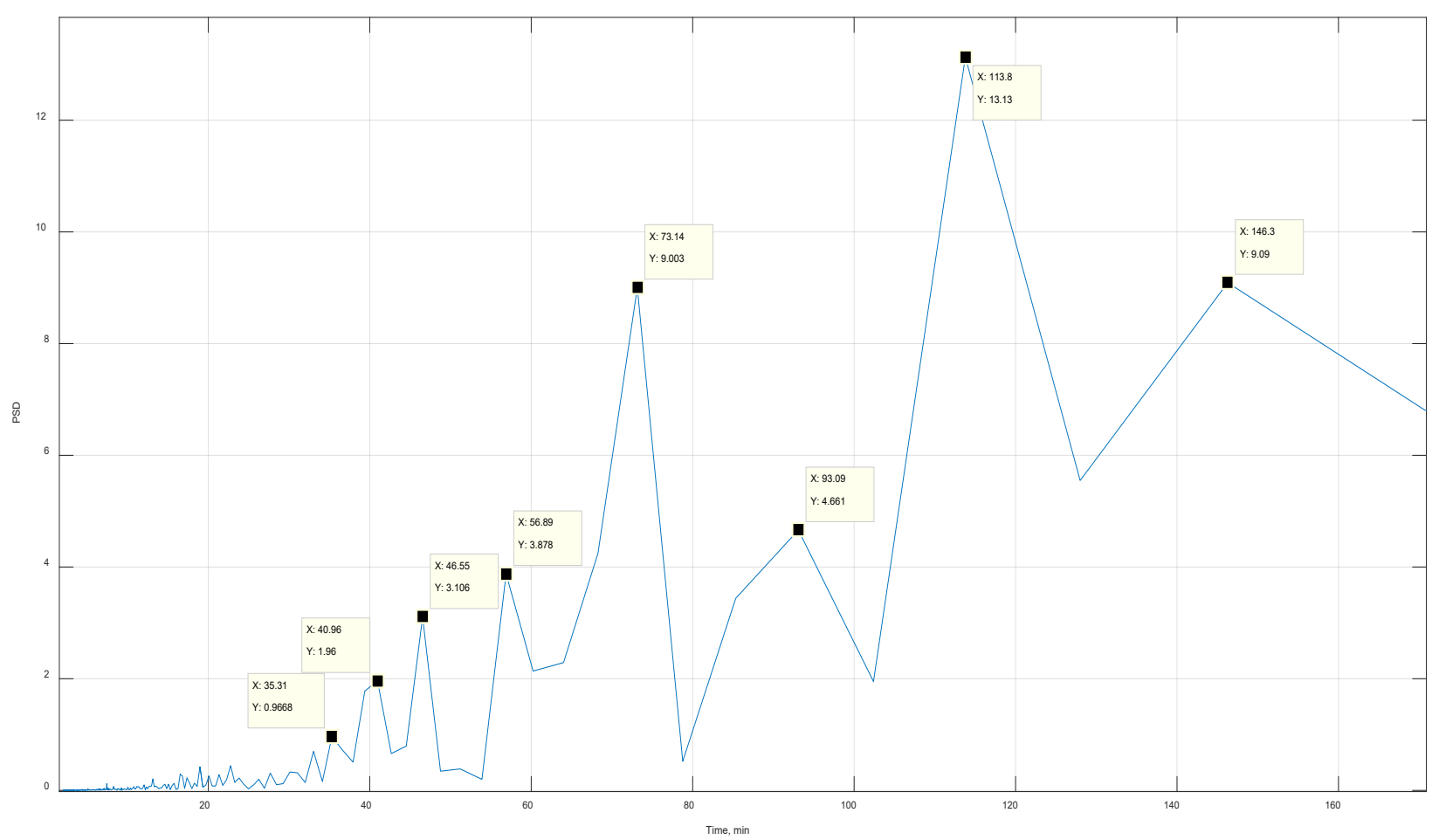

Fig. 2. Power spectrum of the rats of group III temperature fluctuations 


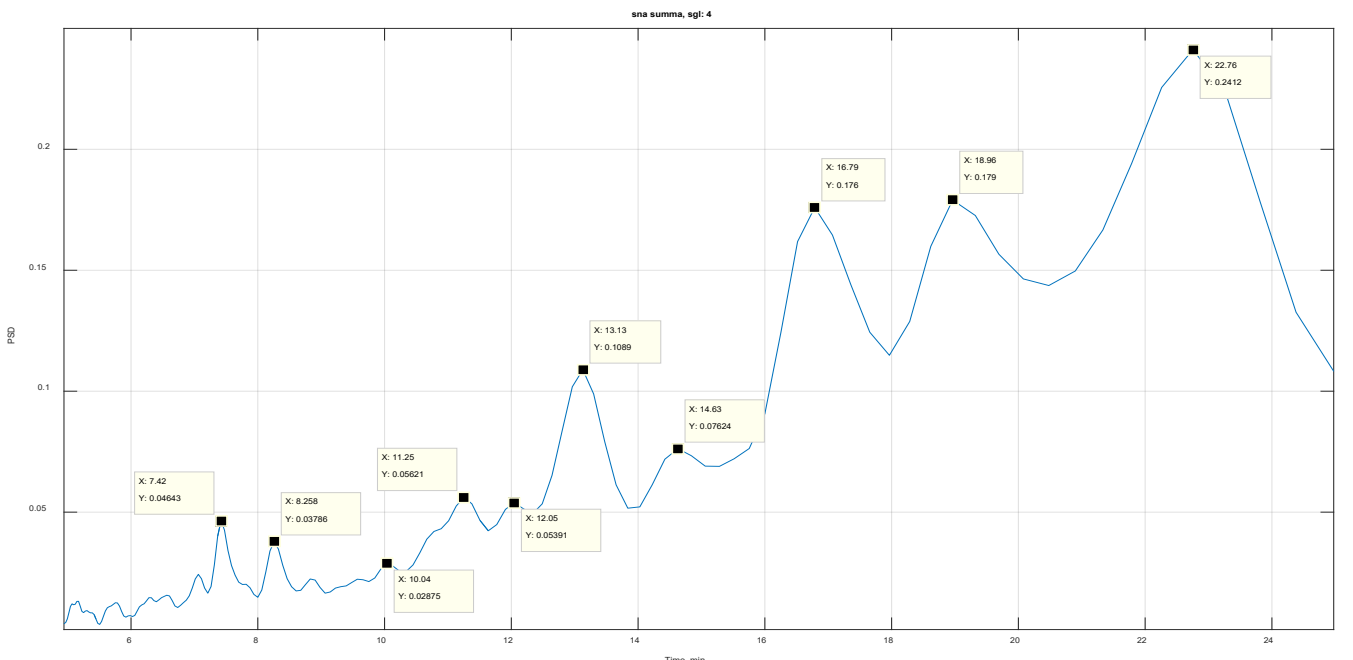

Fig. 3. Enlarged part of the power spectrum from Fig. 2 in the period range of up to $24 \mathrm{~min}$

Table 1 [4-5] contains (a bracketed number corresponds to a line number): (1) - a number of a period; (2) - averaged values of periods found in time series of the alfa-decay fluctuations; (3) - modes of the Earth natural oscillations; (4) - numerical values of the appropriate modes of the Earth natural oscillations; (5) - periods found in the averaged spectrum of the starling temperature fluctuations; (6) - rats I; (7) - rats II; (8) - rats III. Note that the maximal period of the classical Earth natural oscillations corresponds to the main mode of the spheroidal oscillations ${ }_{0} \mathrm{~S}_{2}$ (the averaged value is 53.9 min). For these reasons, lines (3) and (4) of the continued Table 1 are replaced with a single line containing the so called long-wave or seismogravitational natural oscillations of the Earth [8-9].

Table 1. Average values of the periods in the power spectra of the temperature fluctuations and alpha-decay rate fluctuations

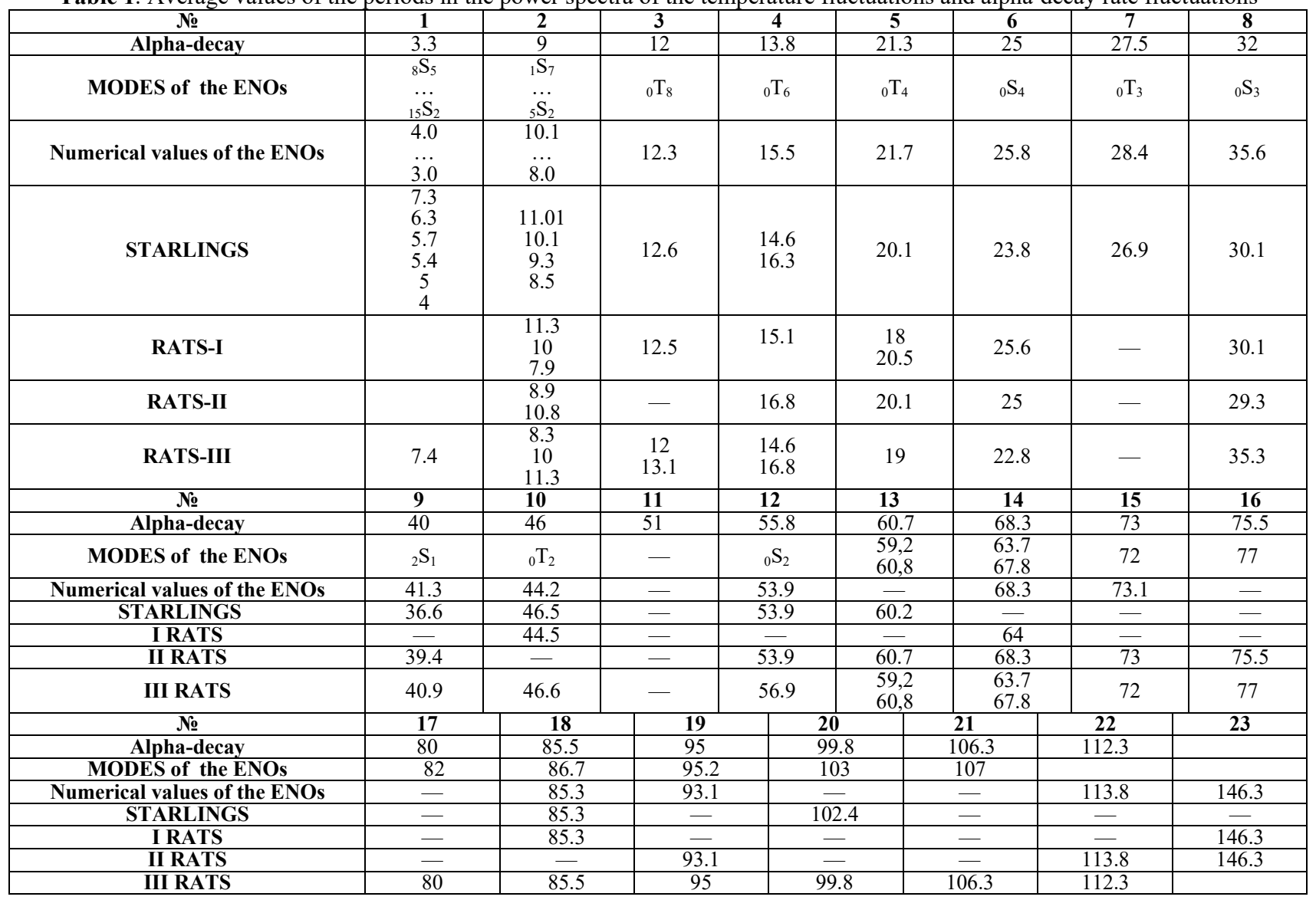

A number of the modes of the Earth natural oscillations grows exponentially with the growth of their frequency. Therefore, the columns number 1 and 2 contain some multitude of the periods. For example, the periods included into the column №2 have 20 modes of natural 
oscillations $(7.97 \mathrm{~min} \ldots 10.1 \mathrm{~min})$, while the periods included into the column №1 have 164 types of the oscillations ( $3 \mathrm{~min} \mathrm{...4} 4 \mathrm{~min}$ ) [10]. Accuracy of the periods determination in the case of the radioactive decay, line (2), is $\pm 1 \mathrm{~min}$, hence comparison of the periods from 1 and 2 in the terms of the present study is senseless, it needs some following investigations with the improvement of the time resolution as the main purpose.

The second distinctive feature of the ENOs is that each mode of the natural oscillations is, in fact, a multiplet, that is, some set of the periods. For example, several periods - 51.5, 52.8, 53.8, 55.2, 56.3 and 58.2 min. correspond to the main mode of the spheroidal oscillations, while Table 1 contains their averaged value, $53.9 \mathrm{~min}$. Therefore, in this case, coincidence of frequencies is rather a crossing of the appropriate sets.

\section{Conclusion}

Important to note that the spectrum, which found in this work coincides with a previously revealed spectrum of the periods, obtained with the use of local fractal analysis by the all permutations method in the course of studies of time series of the alpha decay rate fluctuations. Despite different methods of the experimental data processing used in this work and in work [3-4] (spectral analysis based on Fourier transform and local fractal analysis by the all permutations method), as well as different raw experimental data (time series of the temperature fluctuations in the starlings and rats and noise-like time series of alpha-decay rate fluctuations), the same spectrum of periods was obtained.

In our opinion, the results of the analysis of the time series in the starlings and rats temperature fluctuations presented in Table 1 confirm the hypothesis of the universal character of the spectrum observed initially in the time series of the fluctuations of the radioactive decay rate.

The results of this work can have various practical applications [11-13].

\section{References:}

[1] V.A. Panchelyuga, M.S. Panchelyuga Fractal Dimension and Histogram Method: Algorithm and Some Preliminary Results of Noise-Like Time Series Analysis // Biophysics, 2013, Vol.58, No.2, pp.283289.

[2] V.A. Panchelyuga, M.S. Panchelyuga Local fractal analysis of non-stationary time series by all permutations method // Hypercomplex numbers in geometry and physics, 2014, Vol.11, No.1(21), pp.107-133.

[3] Panchelyuga V.A., Panchelyuga M.S. Local Fractal Analysis of Noise-Like Time Series by the AllPermutations Method for 1-115 min Periods // Biophysics, 2015, Vol. 60, No. 2, pp. 317-330.

[4] V.A. Panchelyuga, M.S. Panchelyuga Some preliminary results of local fractal analysis of noiselike time series by all permutations method for periods 1-120 min // Hypercomplex numbers in geometry and physics, 2014, Vol.11, No.1(21), pp. 134-156.
[5] V. S. Martynyuk Ultradian (Intradiurnal) Geo- and Heliophysically Significant Periods in Integral Rhythm of Animal Locomotive Activity // Biophysics, 1998, Vol. 43, No. 5, pp.747-753.

[6] T.A. Zenchenko, A.A. Medvedeva, N.I. Khorseva, T.K. Breus Synchronization of heart rate indices of human and geomagnetic field variations in the frequency range of $0.5-3.0 \mathrm{mHz} / /$ Geophysical Processes And Biosphere, 2013, Vol.12, No.4, pp.7384

[7] Kopvillem U.Kh., Sharipov R.Z., Zapolsky A.M., Aizdaycher N.A. Biological detector of weak cosmic fields // Biophysics, 1992, vol.37, No.4, pp.643-646.

[8] Buland R., Berger J., Gilbert F. Observations from the IDA network of attenuation and splitting during a recent earthquake. // Nature, 1979, 277, No.5695. pp. 358-362.

[9] Petrova L.N. Seismogravitational oscillations of the Earth and possible mechanism of their formation // Biophysics, 1992, Vol. 37, No. 3, pp. 508-516.

[10] T.G. Masters and R. Widmer, in Free oscillations: frequencies and attenuations, Ed. by T.J. Ahrens (American Geophysical Union, 1995), pp. 104-125.

[11] Syngaevskaya A.A., Berberova M.A., Derevyankin A.A. Studying the effect of stress on the probability of errors by NPP personnel as part of personnel reliability analysis // CPT-2018: proceedings of the International Scientific Conference. - ProtvinoMoscow: Publishing House. IFTI, p. 282-288.

[12]M.A.Berberova, K.I.Chernyavskii, «Comparative assessment of the NPP risk (on the example of Rostov and Kalinin NPP). Development of risk indicators atlas for Russian NPPs», GraphiCon 2019 Computer Graphics and Vision. The 29th International Conference on Computer Graphics and Vision. Conference Proceedings (2019), Bryansk, Russia, September 23-26, 2019, Vol-2485, urn:nbn:de:00742485-1, ISSN 1613-0073, DOI: 10.30987/graphicon2019-2-290-294, http://ceur-ws.org/Vol2485/paper67.pdf, p. 290-294.

[13]Zakharova A.A., Korostelyov D.A., Fedonin O.N. Visualization Algorithms for Multi-criteria Alternatives Filtering (2019). Scientific Visualization 11.4: 66 - 80, DOI: 10.26583/sv.11.4.06

\section{About the authors}

Diatroptov Mikhail Evgen'evich Dr.habil. (Biology), senior research scientist in A.N. Severtsov Institute of Ecology and Evolution, RAS, E-mail: diatrom@inbox.ru

Panchelyuga Victor Anatolyevich, PhD (Physics and Mathematics), senior scientist in the Institute of Theoretical and Experimental Biophysics, RAS E-mail: victor.panchelyuga@gmail.com.

Panchelyuga Maria Sergeevna, scientist in the Institute of Theoretical and Experimental Biophysics, RAS E-mail: victor.panchelyuga@gmail.com.

Seraya Olga Yuzefovna, junior scientist in the Institute of Theoretical and Experimental Biophysics, RAS E-mail: olgaseraya@gmail.com 